\title{
Images of septic thromboembolism from superior vena cava to lung
}

\author{
Deng-Wei Chou ${ }^{1,2} \cdot$ Shu-Ling $\mathrm{Wu}^{3}$
}

Received: 31 August 2015/Accepted: 8 September 2015/Published online: 24 September 2015 (C) SIMI 2015

\begin{abstract}
A 68-year-old woman with end-stage renal disease was started on regular hemodialysis with a tunneled cuffed catheter through the right internal jugular vein for 2 months. She entered our emergency department with complaints of fever, shortness of breath, and chest pain of 2 days duration. On arrival, she was febrile, tachycardic, tachypneic, and had a normal blood pressure. No erythema, swelling, heat, or tenderness was observed at the insertion site of the dialysis catheter. No abnormal breath sounds or audible heart murmurs were detected. Oxygen saturation was $88 \%$ while breathing room air. The electrocardiogram revealed sinus tachycardia. Laboratory examination revealed leukocytosis, thrombocytopenia, and elevated C-reactive protein. An initial chest radiograph (Fig. 1) showed multiple ill-defined opacities in the right lung and the tip of the dialysis catheter was placed in the right atrium (RA). Based on the clinical manifestations and chest radiographic findings, a dialysis catheter-related infection with septic pulmonary embolism (SPE) was suspected. The patient was treated with empirical vancomycin. Contrastenhanced computed tomography (CT) (Fig. 2a-c) performed after dialysis catheter removal revealed that the thrombi were in the superior vena cava (SVC), RA, right
\end{abstract}

Deng-Wei Chou

choudw@gmail.com

Shu-Ling Wu

chwu1898@yahoo.com.tw

1 Department of Critical Care Medicine, Tainan Municipal Hospital, Tainan, Taiwan, ROC

2 Department of Nursing, Chung Hwa University of Medical Technology, Tainan, Taiwan, ROC

3 Department of Long Term Care, Chung Hwa University of Medical Technology, Tainan, Taiwan, ROC ventricle (RV), and right interlobar pulmonary artery branch. CT findings on lung window setting (Fig. 3a-d) showed different types of SPE. A transthoracic echocardiography revealed a $45 \times 26 \mathrm{~mm}$ thrombus within the RA, which was attached to the wall of the atrium, and protruded into the RV (Fig. 2d). The RA and RV were of normal size. Mild tricuspid regurgitation and mild pulmonary hypertension (estimated pulmonary artery systolic pressure $32 \mathrm{mmHg}$ ) were detected. Anticoagulation therapy was initiated. The catheter tip culture and three pairs of blood cultures were positive for oxacillin-sensitive Staphylococcus aureus. Based on radiographic evidence of thrombi and positive blood culture results, the diagnoses of septic thrombophlebitis of the SVC, septic thrombosis of the RA with pulmonary embolism (PE), and SPE were confirmed.

In a previous study, approximately $12.5 \%$ of patients exhibit thrombus at the tip of catheter within 6-8 weeks of catheter insertion, and all thrombi are found in those patients with the catheter tip position in the RA, in contrast to none in those with the catheter tip position in the SVC [1]. The likely mechanism of thrombus formation at this location is related to friction of the catheter on the atrial endocardium $[2,3]$. The endothelial damage induced by trauma results in platelet aggregation and thrombus formation [4]. An adherent infected thrombus in the RA may become dislodged, and lead to PE and SPE. Great diversity of SPE can be found in CT findings, including a feeding vessel sign, nodules with or without cavities, pleural effusions, peripheral wedge-shaped opacities, patchy ground-glass opacities, lung abscesses, and focal consolidations [5].

A chest radiograph can assist in diagnosis of this disease, as seen in the present case. A possible diagnosis of dialysis catheter-related SPE from a chest radiograph may 


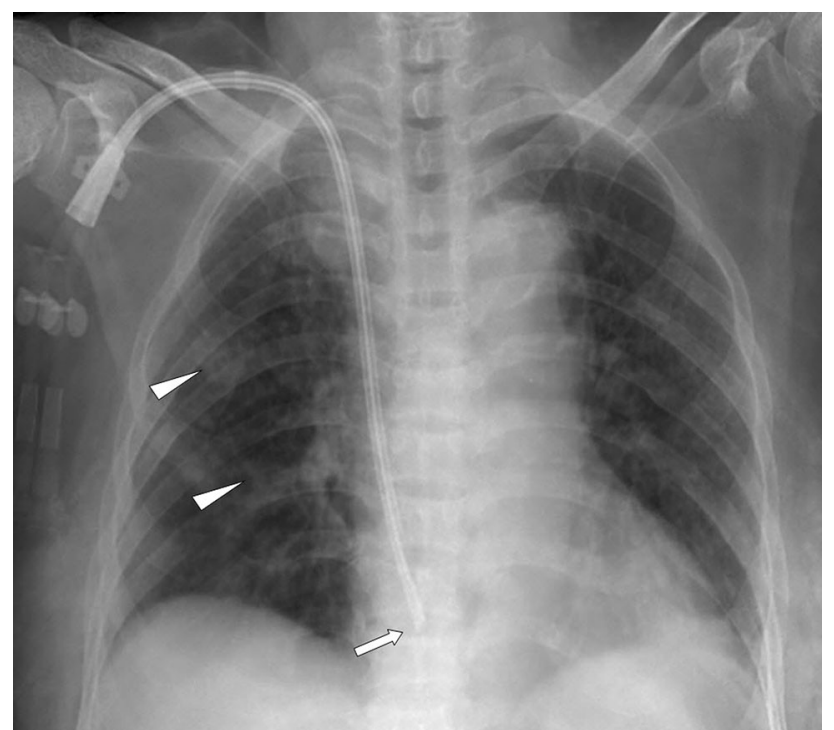

Fig. 1 A chest radiograph showing multiple ill-defined opacities (arrowheads) in the right lung. The tip (arrow) of the dialysis catheter is placed in the right atrium (RA) direct the physician to search for right heart thrombus, particularly in a patient with the tip of the dialysis catheter placed in the RA. A CT scan should follow the chest radiograph to identify $\mathrm{PE}, \mathrm{SPE}$, and the presence of thrombosis in the SVC or RA. An echocardiography (transthoracic or transesophageal) should be performed to detect and assess right heart thrombus in such a case. An echocardiography can define the size, mobility, and site of attachment of right heart thrombus. The present case is valuable as a reference because the thrombi in the SVC, right heart, and interlobar pulmonary artery branch are clearly identified, and protean presentations of SPE are visualized.

\section{Compliance with ethical standards}

Conflict of interest The authors declare that they have no conflict of interest.

Statement of human and animal rights All procedures performed in studies involving human participants were in accordance with the ethical standards of the institutional or national research committee
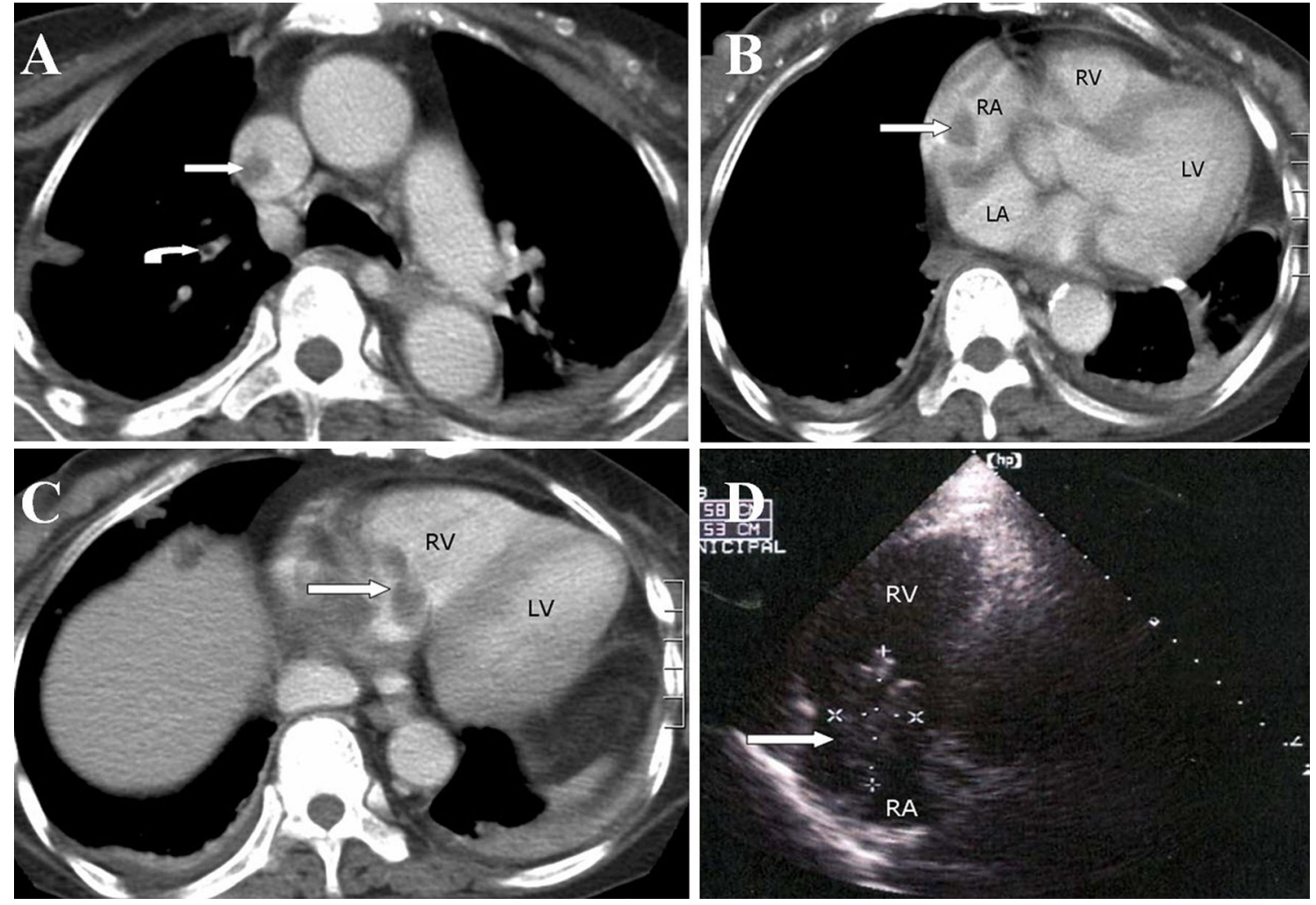

Fig. 2 Contrast-enhanced CT images showing that filling defects are in a the superior vena cava (arrow), right interlobar pulmonary artery branch (curved arrow), b RA (arrow), and c right ventricle (RV) (arrow), which are suggestive of thrombi. d Two-dimensional transthoracic echocardiography (modified apical 4-chamber view) shows a $45 \times 26 \mathrm{~mm}$ thrombus (arrow) within the RA, which is attached to the wall of the atrium, and protrudes into the RV. $L A$ left atrium, $L V$ left ventricle 


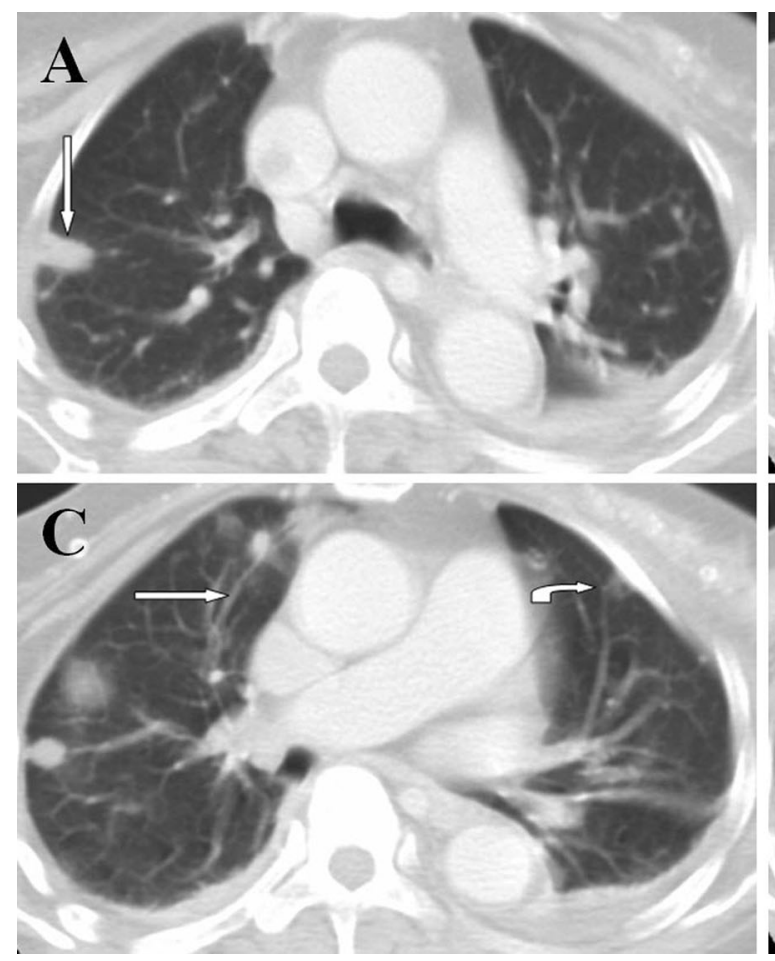

Fig. 3 CT findings on lung window setting show a a peripheral wedge-shaped opacity abutting pleural surface (arrow), b a focal consolidation with air-bronchogram (arrow), c a nodule with a

and with the 1964 Helsinki Declaration and its later amendments or comparable ethical standards. This article does not contain any studies with animals performed by any of the authors.

Informed consent Informed consent was obtained from all individual participants included in the study.

\section{References}

1. Gilon D, Schechter D, Rein AJ, Gimmon Z, Or R, Rozenman Y, Slavin S, Gotsman MS, Nagler A (1998) Right atrial thrombi are related to indwelling central venous catheter position: insights into time course and possible mechanism of formation. Am Heart $\mathbf{J}$ 135(3):457-462

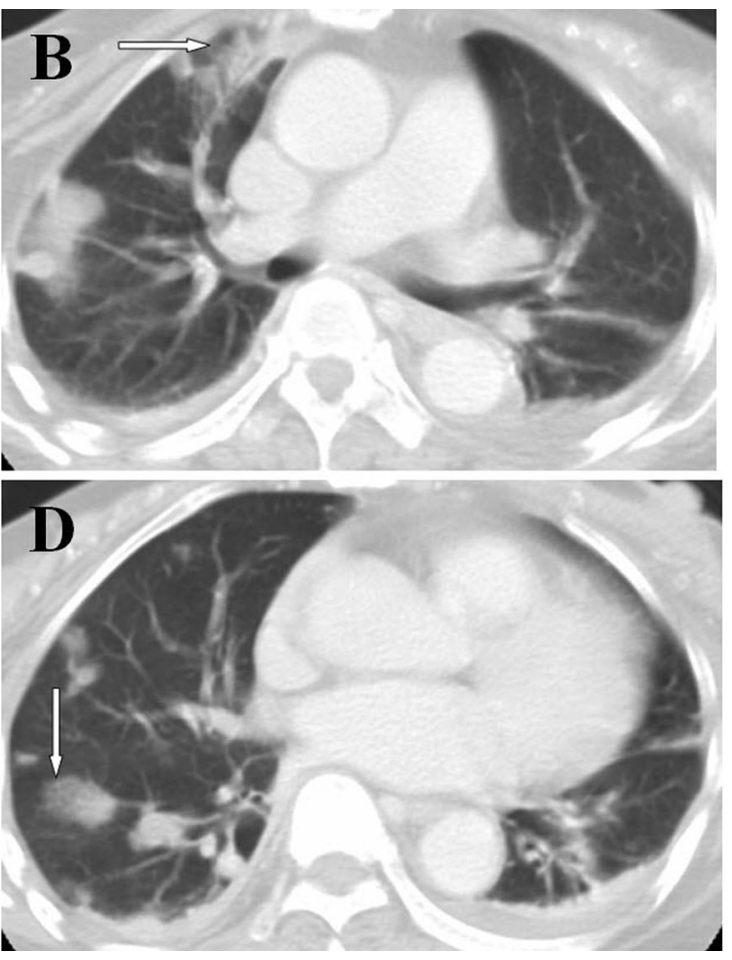

feeding vessel sign (arrow), a nodule with cavity (curved arrow), $\mathbf{d}$ a focal ground-glass opacity (arrow), and left pleural effusion

2. Ghani MK, Boccalandro F, Denktas AE, Barasch E (2003) Right atrial thrombus formation associated with central venous catheter utilization in hemodialysis patients. Intensive Care Med 29(10):1829-1832

3. Fuchs S, Pollak A, Gilon D (1999) Central venous catheter mechanical irritation of the right atrial free wall: a cause for thrombus formation. Cardiology 91(3):169-172

4. Abid Q, Price D, Stewart MJ, Kendall S (2002) Septic pulmonary emboli caused by a hemodialysis catheter. Asian Cardiovasc Thorac Ann 10(3):251-253

5. Chou DW, Wu SL, Chung KM, Han SC (2015) Septic pulmonary embolism caused by a Klebsiella pneumoniae liver abscess: clinical characteristics, imaging findings, and clinical courses. Clinics (Sao Paulo) 70(6):400-407. doi:10.6061/clinics/ 2015(06)03 